\title{
Acción conjunta y conjunción de esfuerzos en las Fuerzas Armadas
}

\section{Francisco Laguna Sanquirico}

Arbor CLXV, 651 (Marzo 2000), 397-414 pp.

\section{Introducción}

La conocida historia de aquel padre que para aleccionar a sus hijos sobre la necesidad de permanecer unidos les dio a quebrar primero una rama y después un mazo, demostrándoles que si bien era fácil romper la primera no podían hacer lo mismo con el conjunto, sirve sin duda para ilustrar las ventajas de actuar en equipo, por encima de las acciones individuales a las que el hombre en último término tiende.

Sin embargo, a pesar de que aprendió desde los primeros tiempos, posiblemente al ver como cazaban algunos animales, que para sobrevivir era preciso conjuntar su esfuerzo con el de otros y superar su individualismo, no ha desaparecido en el fondo del ser humano la inclinación a mantener sus diferencias y cierto distanciamiento con el resto de los grupos. Se puede afirmar que en el hombre, y por extensión en la sociedad, coexisten y se contrapesan las tendencias a lo colectivo $\mathrm{y}$ a lo individual, a unirse a otros para actuar y a considerar que con ello peligra su autonomía.

Los ejércitos, de igual modo que el resto de la sociedad de la que forman parte, viven en el marco de esta tensión, pero no sería correcto basar exclusivamente en esta circunstancia la problemática actual de los Ejércitos en relación a lo que se denomina «acción conjunta». Por mucho que se encuentren en ella los fundamentos de las modernas doctrinas que regulan las operaciones militares, no se debe simplificar hasta el punto de prescindir de cuanto se ha ido configurando a través de la historia. 
Si bien son muchos los ejemplos que demuestran que, desde la antigüedad, los grandes capitanes buscaron la victoria en la conjunción de los esfuerzos de sus tropas, la realidad es que considerar la acción conjunta como un criterio fundamental de la táctica, la estrategia o la logística, es mucho mas reciente.

Por otro lado, las Fuerzas Armadas representan bastante mas que un cierto número de unidades del Ejército de Tierra, de la Armada o del Ejército del Aire. En su condición de Instituciones forman parte del conjunto de la sociedad y, en consecuencia, existe una permanente corriente de ideas y de percepciones entre las Fuerzas Armadas y el resto de la nación. De esta osmosis se deduce que el tema que nos ocupa puede estudiarse enmarcado en dos contextos distintos: el que se refiere al propio ser de las Fuerzas Armadas, su estructura, sus misiones y sus normas de actuación, y el que se refiere al objeto de la Defensa, o lo que es lo mismo, a su influencia sobre otros sectores de la sociedad y sobre sus pautas de conducta, por la necesidad que tienen los pueblos de prever la defensa de sus intereses.

\section{La conjunción de esfuerzos en el propio «ser» de las FAS}

Al concluir la II Guerra Mundial, el Presidente norteamericano Eisenhower, afirmó en el Congreso de los EE.UU. que «las guerras separadas en tierra, mar o aire se habían acabado para siempre». Mas allá de la valoración que pueda hacerse sobre estas palabras por el lugar en que fueron pronunciadas, su importancia reside en que quien las dijo había ejercido el mando en la mayor operación combinada y conjunta de la historia.

Como se ha indicado, el pensamiento militar desde siglos atrás ya había planteado la necesidad de aunar esfuerzos. Así en el conocido texto sobre el arte de la guerra de Sun Tzu se dedican dos apartados a este tema, uno al «apoyo íntimo que debe existir entre las diferentes partes de un ejército» y otro «al enlace entre las armas y de la unión de los combatientes» ${ }^{1}$.

Siglos después Clausewitz en su tratado «De la Guerra» ${ }^{2}$, dedicó también dos capítulos a la «reunión de fuerzas en el espacio» y a la «reunión de fuerzas en el tiempo».

Hoy pueden parecer simples y hasta pobres tales planteamientos pero no lo son tanto si se sitúan en un contexto en el que los combates se libraban en base a la acción individual de los combatientes y, todo 
lo mas, en la adecuada colocación de las tropas para adoptar el orden de batalla. Sin embargo no se debe negar la diferencia que existe entre estas recomendaciones a la coordinación de esfuerzos y al concepto moderno de acción conjunta. Este no puede entenderse simplemente como una prolongación de aquél, sino que representa un salto cualitativo.

Salto cualitativo provocado fundamentalmente por los cambios profundos en el entorno estratégico y de seguridad, los cambios sociales $\mathrm{y}$, sobre todo, por los tecnológicos ${ }^{3}$. Todo hace pensar que en el próximo siglo será una realidad la afirmación del general Eisenhower y así lo entienden las Fuerzas Armadas de las naciones mas desarrolladas, que están revisando sus conceptos estratégicos y las estructuras de mando para las acciones del próximo siglo.

Mas allá de la acción puramente militar también existen antecedentes de la necesidad de unificar esfuerzos y responsabilidades. Así el Rey Fernando el Católico nombra a Gonzalo Fernández de Córdoba Capitán General de mar y tierra en la segunda campaña de Italia, perfeccionando la situación anterior en la que el Gran Capitán tenía sólo el mando de las fuerzas de tierra mientras el Conde de Trivento lo ejercía sobre la escuadra de galeras de Sicilia. Generalizando puede decirse que en aquel período no existía unidad de mando a nivel estratégico. Los virreyes o cargos equivalentes, eran los responsables políticos y en cierta medida tenían potestad sobre las unidades de tierra y sobre la marina, pero normalmente la acción militar se desarrollaba en uno u otro escenario y todo lo más se ordenaba el apoyo mutuo para una operación concreta.

Estudiar en detalle la evolución de estos acontecimientos y su incidencia sobre la doctrina militar, nos apartaría sin duda del objeto de estas reflexiones. Quede, sin embargo, apuntada la idea de que la progresiva complejidad en la orgánica de los Ejércitos y la de los medios de combate ha ido configurando un planteamiento diferente de las operaciones militares. En el último siglo esta evolución se une a la que sufren las estructuras de la Administración del Estado, en las que se hace evidente que el incremento de nuevas tareas y misiones, unido a la modernización de los medios disponibles, obliga a una mayor coordinación de los organismos responsables de cada una de las áreas.

Limitándonos al caso de España puede ser útil para comprender el estado actual de la acción conjunta en las Fuerzas Armadas, recorrer, aunque sea de forma esquemática, los acontecimientos y disposiciones legales mas recientes que la fundamentan, unos referidos a la orgánica y otros a la estrategia propiamente dicha. 
Incidencia de la importancia de la conjunción de esfuerzos en la orgánica de Defensa

Para entender la evolución de las Fuerzas Armadas en este tema es conveniente precisar algunos términos que en ocasiones se emplean de forma indiscriminada. Durante siglos la palabra «ejércitos» se ha interpretado como una definición del total de las Fuerzas Armadas, pero en sentido estricto se refiere exclusivamente al Ejército de Tierra. Por su parte al conjunto de las fuerzas navales se ha denominado genéricamente como «la Marina» o la Marina de guerra pero hoy en casi todas las naciones se las conoce como «Armada».

Entre Ejército y Armada han existido, desde antaño y en todos los países, notables diferencias, tanto en su organización, como en sus tradiciones y en su doctrina de empleo. Como es lógico, la organización de las fuerzas aéreas como tercer ejército, mucho mas moderno, no tiene tantos condicionantes, pero la importancia de su participación en los últimos conflictos bélicos está ya generando rasgos diferenciales, tanto respecto al Ejército como a la Armada. Existe por tanto, históricamente, una diferenciación entre unos y otros que ha llevado en algunos casos a la rivalidad, o cuando menos, a mantener posturas bastante distanciadas sobre los problemas estratégicos.

A nivel político se planteó una situación similar. A los Secretarios de Estado del antiguo régimen les sucedieron dos Ministerios: el de la Guerra y el de Marina. Solamente durante la guerra civil 1936-39 se unificaron en un solo Organismo, pero este hecho indicó que en la guerra moderna ya no era posible mantener por separado la responsabilidad de ambas fuerzas. Terminada la contienda se volvió a dos Ministerios, a los que se sumó el del Ejército del Aire.

Simultáneamente, aunque con nivel orgánico inferior, se creó el Alto Estado Mayor como órgano de coordinación. En realidad se trataba de institucionalizar el Estado Mayor del Jefe del Estado mas que de crear un órgano que cubriera una necesidad sentida por las Fuerzas Armadas. Como consecuencia resultó poco operativo en orden a desarrollar con eficacia la tarea de coordinación entre los Ejércitos.

Con todo, la necesidad de lograr una mayor coordinación quedaba planteada, lo que posibilitó, de forma similar a lo que sucedía en otros países del entorno, la creación, en 1969, del Centro Superior de Estudios de la Defensa Nacional (CESEDEN), con la misión de estudiar, entre otros temas relacionados con la defensa, la doctrina para el desarrollo de la acción conjunta. En concreto uno de los temas que se 
le encomendaron fue el de la oportunidad de un Ministerio de Defensa en sustitución a los tres existentes.

En febrero de 1975 se dio un nuevo paso con la creación de la Junta de Jefes de Estado Mayor como órgano de mando operativo y en abril de 1977, por RD.1558/77, el Ministerio de Defensa Nacional, transformándose los antiguos Ministerios, en los Estados Mayores de cada uno de los Ejércitos. Desde el punto de vista de la orgánica este paso constituyó, en lo que se refiere a la estructura de la Defensa, el cambio mas importante de los últimos tiempos, con lo que así mismo se confirmaba la tesis de que las operaciones militares del futuro tendrían, en la mayoría de los casos, el carácter de acciones conjuntas.

Estos avances se vieron refrendados con la promulgación de la Ley Orgánica 6/1980 de «Criterios Básicos de la Defensa y de la Organización Militar», en la que se fijaban los diferentes ámbitos de competencias en relación a la Defensa y al mando operativo de las Fuerzas. El conjunto de estas leyes puede tacharse de excesivamente prudente, por cuanto procuraba respetar al máximo las tradiciones y una cierta autonomía para cada Ejército, pero a la vez hacía hincapié en la necesidad de conjugar los esfuerzos, tender a una mayor coordinación y se apuntaba a una futura integración de todo aquello que fuera común y no tuviera razones de peso que justificaran aquella autonomía.

En este sentido es significativo que en el Real Decreto 1883/96, de 2 de agosto, "de la estructura básica del Ministerio de Defensa», se define al Jefe del Estado Mayor de la Defensa como «la autoridad militar a través de la cual el Ministro ejerce su autoridad para ordenar, coordinar y dirigir la actuación de las Fuerzas Armadas en el cumplimiento de las misiones operativas derivadas del Plan Estratégico Conjunto» (art. 7). Y le señala entre otras las siguientes funciones:

- Proponer el Plan Estratégico Conjunto

- Proponer prioridades

- Establecer la Doctrina para la acción conjunta

- Proponer la unificación de Servicios...

- Coordinar la cooperación con otras Fuerzas Armadas

Así mismo se estructura, como principal órgano auxiliar de mando del Jefe del Estado Mayor de la Defensa, un Estado Mayor Conjunto y se posibilita la creación de Mandos unificados cuando las circunstancias lo aconsejen. Resulta significativo, sin embargo, que a pesar de esta normativa sobre la estructura de los Mandos, en los últimos años solamente se haya organizado como tal el Mando Unificado de 
Canarias, que además, ha sido recientemente suprimido al carecer de una misión estrictamente necesaria.

A pesar de la evidente relación que puede existir con esta evolución de la orgánica, es conveniente dejar al margen la incidencia que tuvo, en el período de la transición, el proceso político en cuanto a la subordinación de las Fuerzas Armadas al poder «civil». Aunque de algunos textos se deduce la necesidad de que un tema tan vital para la nación como la Defensa tenga en último término un responsable único, que no puede ser otro que quien dirige la política nacional, mezclarlo con el de la «acción conjunta», que corresponde a otro nivel, puede dar lugar a confundir las ideas.

En la legislación vigente, desde la misma Constitución a las leyes posteriores que las desarrollan, queda claro que el Gobierno es quien dirige la Administración civil y la militar y en línea con este criterio la citada LO. 6/80 contempla a la Junta de Defensa Nacional, órgano superior y consultivo del Gobierno, en el capítulo dedicado a los Órganos Superiores de la Defensa Nacional.

\section{Evolución de la Doctrina en relación a la «acción conjunta»}

Si nos remontamos al siglo en el que, como soporte del Estado moderno, se organizaron los ejércitos permanentes, el precursor de esta doctrina fue Carlos I, quien afirmaba que era necesario tener una «concepción unificadora imperial» con un solo Mando. Pero ya se ha indicado que, en rigor, las actuales teorías estratégicas no son equiparable a las del siglo XVI, y ha sido en los últimos 50 años cuando se ha dado un salto cualitativo en las doctrinas de empleo de los ejércitos.

Mas próximas están la «Doctrina para el empleo de las Armas y Servicios» de 1924 y el Decreto de la Presidencia del Gobierno publicado en 1942. En la primera se incluye entre los principios fundamentales del arte de la guerra «la acción de conjunto», concepto que se mantiene en las redacciones posteriores y que termina desapareciendo en la de 1996; en la de 1998,al seguir otro esquema, suprime el capítulo dedicado a los «principios». En cambio, en este último texto si se tratan expresamente las «actividades conjuntas» sobre las que se dice que « la ejecución de las operaciones en todo el campo de batalla exige la integración de las energías de la nación materializadas por la cooperación entre los distintos Ejércitos, así como la de las Organizaciones gubernamentales de carácter civil» ${ }^{4}$.

En 1942, cuando no había aún finalizado la Guerra Mundial, se publicaba un Decreto en el que se fijaban normas para «el Mando conjunto» 
y la sucesión del mismo en los Ejércitos de Tierra, Mar y Aire. Estos documentos, junto a la «Doctrina» de 1924, pueden considerarse como los antecedentes formales de la «acción conjunta» en nuestro país.

Como elemento más permanente en el desarrollo de estos conceptos ha de citarse al CESEDEN, que ya se ha indicado tenía como una de sus misiones principales su estudio. En la organización del Centro se incluyó la Escuela de Estados Mayores Conjuntos con el objeto de que entre los oficiales de Estado Mayor de cada Ejército, se fuera formando un núcleo que potenciara esta línea de actuación y estuviera en condiciones de ocupar los puestos, cada vez mas numerosos, de los organismos que tenían estas funciones.

Resultado de los primeros estudios fue la redacción de un Proyecto de Doctrina de Acción Unificada. Aunque el documento estuvo aprobado, con carácter provisional, nada menos que en 1976, hasta la fecha no ha sido factible publicar un texto definitivo. En la actualidad, el proyecto ha quedado aparcado, no tanto por la dificultad de llegar a un texto que conjugara las peculiaridades operativas de los tres Ejércitos y recibiera su respaldo, como por el hecho de que se ha considerado mas oportuno adoptar la Doctrina OTAN que está ampliamente contrastada, con lo que además se facilita nuestra integración en dicha Organización.

Por otro lado, la OTAN ha aprobado recientemente el concepto de «acción combinado-conjunta» para las nuevas misiones, dando así un impulso a lo que, desde todos los puntos de vista, se considera fundamental para la eficacia de las Fuerzas Armadas modernas.

La doctrina aliada para operaciones conjuntas, aunque está pensada para las misiones OTAN, puede aplicarse, sin mayor problema, a otras Fuerzas multinacionales o a las de países miembros de la Unión Europea Occidental, como es el caso de España, y en ella se incluye una gran variedad de operaciones distintas a las clásicas de guerra. Tiene, en consecuencia, gran aplicación para las intervenciones de ayuda humanitaria o para las operaciones de Paz de las Naciones Unidas. En este sentido hay que señalar que el capítulo 21 se refiere a la «cooperación cívico-militar» (CIMIC) en el que se fijan como principios a tener en cuenta: la misión, la responsabilidad de mando y la continuidad y consistencia de la política.

\section{Definiciones}

A lo largo de estas consideraciones se han empleado casi indistintamente los términos de «acción unificada», «acción conjunta» y «acción 
combinada». Si bien se trata de conceptos muy próximos, existen diferencias que conviene precisar. El término «acción unificada» fue el empleado para la redacción por el CESEDEN del documento ya citado y durante unos años se utilizó como referencia a las operaciones militares en las que, bajo un solo mando, intervenían unidades de mas de un Ejército. Una definición mas completa es la que describe la acción unificada como «aquella que desarrolla el Mando en sus mas altos escalones, a nivel de decisión, para concertar el planeamiento y la coordinación de los distintos Ejércitos que actúan con una finalidad común ${ }^{5}$.

En textos posteriores se fueron introduciendo los términos de «Operaciones conjuntas» y «Operaciones combinadas», que son los que mayoritariamente se utilizan en los documentos de las Fuerzas Armadas de nuestro entorno.

«Operaciones conjuntas» son aquellas en las que bajo un mando único, intervienen unidades de mas de un Ejército, pero de un mismo país, mientras que se califican de «Combinadas» aquellas en las que intervienen fuerzas de varios países. Existe por lo tanto una diferencia substancial entre ambos conceptos, ya que si el primero apunta hacia la necesidad de emplear conjuntamente todos los esfuerzos de que dispone una nación para defenderse, el segundo se orienta a la moderna teoría estratégica de la "defensa colectiva», esto es, a que en el futuro, cada vez más, la seguridad internacional se apoyará en la actuación coordinada de varias naciones.

Por su parte los Reglamentos OTAN definen la «Operación aliada conjunta» como aquella que llevan a cabo fuerzas pertenecientes a dos o mas países de la OTAN, en las que participan elementos de mas de un Ejército y distingue su aplicación en los niveles estratégicos, operativos y tácticos. En todos ellos se destaca como principio fundamental la unidad de mando, reforzado con el concepto de continuidad en el ejercicio del mando: «quien planea ha de ejecutar».

Esta definición, mas amplia que la que recoge la antes citada «Doctrina para el empleo de las Armas y Servicios del Ejército de Tierra», puede aplicarse a toda clase de operaciones. Si a ello se añade el «nuevo concepto estratégico» recientemente aprobado en la Cumbre de Bruselas de 1994, en la Cumbre de Roma y en la Reunión Ministerial de 1996, parece evidente que, en el futuro inmediato, las Fuerzas Armadas de los países de la Alianza han de orientarse hacia esta nueva concepción de las Operaciones militares.

Sin que se pueda afirmar que vayan a desaparecer las amenazas singulares a los intereses propios de una nación (para las que también 
ha de estar preparada), en el horizonte próximo, los riesgos y las amenazas afectarán a mas de un país y, en todo caso, los Pactos y los Acuerdos van a caracterizar las intervenciones en defensa de los intereses nacionales.

Este modo de plantear la defensa afecta tanto al campo de la orgánica como al operativo de los Ejércitos. A la orgánica porque exige unas estructuras mas flexibles con capacidad de integrar a otras Unidades o de formar parte de una Unidad superior. En la medida que las operaciones combinadas se llevan a cabo con Unidades pertenecientes a distintos ejércitos, es evidente que se hace necesario aproximar, en todo lo posible, equipamiento, organización y normas de actuación.

En cuanto a lo operativo, se hace indispensable la existencia de una doctrina común de empleo de las Fuerzas, superando recelos y susceptibilidades, puesto que de otro modo no será posible llegar a una acción combinada. Todo lo mas se lograría la cooperación o el apoyo. Conviene recordar que las Alianzas que se formaron en otros tiempos se realizaban para enfrentarse a un enemigo concreto $y$, en la mayoría de las ocasiones, para una batalla o una campaña determinada. Las Operaciones combinadas que ahora se plantean tienen un mayor alcance en cuanto suponen una concepción nueva de la estrategia para la paz, la seguridad y la defensa.

Consecuencia inmediata de la aplicación de esta doctrina ha de ser, necesariamente, una mayor integración entre los Ejércitos de cada nación. En los últimos años, en España se han dado pasos importantes en el ámbito de la enseñanza y en la estructura de ciertos Órganos comunes. El mismo Ministerio de Defensa supone un avance en relación a los anteriores tres Ministerios. Pero no se puede considerar como suficiente y es de esperar que progresivamente, y de manera cada vez mas acentuada, se vayan integrando los órganos logísticos y se aproxime la formación de los cuadros de mando, primero en el ámbito de cada Ejército y en segundo lugar, en el de los otros dos.

Parece evidente que sería un contrasentido que los Ejércitos de una misma nación no fueran capaces de desarrollar una auténtica política conjunta en los diferentes campos en que realizan funciones similares, y se desarrollara una doctrina de acción combinada con las Fuerzas Armadas de otros países.

Otra consecuencia será la necesidad de que, desde todos los campos, se realice un gran esfuerzo en favor de la interoperatividad. Según el glosario OTAN de definiciones, ésta significa «la capacidad de los sistemas, unidades o Fuerzas cuya organización y relaciones respectivas les permita ayuda mútua que les posibilita operar de común acuerdo». 
La interoperatividad se relaciona corrientemente con el material, pero va mucho mas allá y abarca tanto los procedimientos operativos comunes, la organización similar de las Unidades y de los órganos de mando, como la normalización de los equipamientos ${ }^{6}$. No se trata solo de una cuestión militar sino que alcanza a los mas diversos sectores de la vida. El desarrollo de los medios de comunicación social, la mundialización del comercio y tantos otros fenómenos de la era moderna, imponen la exigencia de normas y sistemas comunes y se oponen a los particularismos sin justificación.

Gran parte de este proceso se debe al extraordinario desarrollo tecnológico del último cuarto de siglo. Los medios de mando y control, las aplicaciones informáticas en los mas diversos campos y la necesaria simplificación en el apoyo logístico, son consecuencias de este progreso. Tanto por eficacia como por economía es necesario lograr la máxima interoperatividad en todos los ordenes y esta exigencia habrá de tener su reflejo no solo en los planes de cooperación internacional sino también en las programaciones de cada nación.

No se debe obviar que en algunos sectores, suscita recelos y hasta rechazo. Al no estar fijados los límites del proceso se entiende que puede existir el riesgo de caer en el extremo de la «unificación», con lo que, en lo multinacional, se perderían muchos de los valores de los Ejércitos menos fuertes y, a nivel nacional, se podrían perder tradiciones y símbolos de cada uno de los Ejércitos (en cada uno de ellos también se teme que puedan perderse rasgos y peculiaridades de las diferentes Armas y Cuerpos).

Es por tanto necesario fijar los conceptos y distinguir lo que significa interoperatividad de lo que sería un proceso de unificación igualatoria. Por lo pronto las funciones y misiones de cada Unidad determinan unas características que no es conveniente perder. Además, la historia ha ido enriqueciendo a los distintos ejércitos con tradiciones y símbolos que no sólo deben mantenerse, sino que es conveniente cultivar y difundir. El combatiente no se motiva por lo que dictan los reglamentos, sino por lo que le impulsan sus convicciones y los símbolos que las representan.

Una última consecuencia del nuevo concepto estratégico es que las Unidades militares habrán de ser mas ágiles y flexibles que las actuales. A partir de la citada Cumbre de Bruselas se consideran como claves, para que puedan ser desarrolladas las operaciones combinado-conjuntas, que las Unidades tengan suficiente movilidad para ser desplegadas rápidamente en el área en la que se necesiten, y que sean flexibles en su organización y medios, para poder atender a las 
diversas misiones que le pueden ser encomendadas. En definitiva que posean lo que hoy se denomina «capacidad de proyección».

Desbordaría el objetivo de este trabajo detallar las consecuencias de ambas características, pero es importante apuntar que son fundamentales la «disponibilidad», en cuanto al despliegue y transporte, y la composición «modular», que permita combinar los medios adecuados para cada caso. Estas y otras características que se podrían añadir, impondrán en los próximos años una importante reorganización en las Fuerzas Armadas, tanto en su estructura y despliegue, como en la dotación de medios y en la formación del personal.

\section{La acción conjunta en relación al «objeto» de la Defensa}

La necesidad de defenderse de las amenazas y de prevenir los riesgos tiene por si mismo un efecto integrador. En la medida que los Estados precisan de unas estructuras estables para la Seguridad y la Defensa, las Instituciones que las mantienen cooperan en este objetivo último de mantener la unidad y favorecer la cooperación entre los distintos sectores de la comunidad nacional.

La paz se ha transformado en uno de los principales anhelos de la humanidad y en lograrlo confluyen los esfuerzos de muy diferentes Organismos, incluidas las Organizaciones no gubernamentales, y por supuesto las Fuerzas Armadas.

En las naciones mas desarrolladas se han superado los tiempos en los que los poderes públicos utilizaban sistemáticamente a los ejércitos para mantener el orden y para frenar los deseos separatistas o de independencia de las comunidades. Hoy, el poder integrador de las Fuerzas Armadas no se deriva tanto de estas intervenciones cuanto del hecho de que la defensa es un bien común y colectivo. No se concibe la defensa de un sector o de una región del territorio sin que se vea implicado el resto.

En este sentido la influencia de las nuevas concepciones de la «defensa colectiva" y la participación en los Organismos internacionales de Seguridad y Defensa, coadyuvan a desarrollar este efecto de cohesión que tienen las Fuerzas Armadas. En los dos últimos siglos se ha pasado de los ejércitos «del Rey», a los ejércitos nacionales, y por último a los multinacionales. Es por tanto una tendencia que colabora en la formación de una verdadera comunidad internacional.

Aunque todavía nos encontremos lejos de este ideal, es significativo que en Europa, continente que se ha formado en base a continuos 
conflictos bélicos, se esté hablando cada vez mas de la «Defensa común». Los primeros objetivos se han planteado en el campo de la economía, especialmente en la moneda única, pero existe una convicción general de que sólo se logrará una unidad suficiente, cuando se logre establecer una política exterior y una política de defensa comunes ${ }^{7}$.

Sobre tres parámetros cabe estudiar el efecto positivo de la Defensa, en orden a la unidad de los diferentes sectores que componen la comunidad nacional. En el de la política, en el de la propia sociedad $\mathrm{y}$ en el de los valores éticos.

\section{Incidencia en el ámbito sociopolítico}

Bastantes politólogos modernos consideran que el proceso de globalización, tantas veces mencionado, está provocando como reacción la aparición con renovada fuerza de los nacionalismos. Estos, que se consideraban fenómenos del pasado o propios de naciones que estrenaban su independencia, hoy aparecen en gran número de Estados modernos y desarrollados, en contra de las teorías de los tratadistas de principios de siglo.

Al margen de la valoración que desde distintos puntos de vista se pueda hacer, es indudable que este cruce de fuerzas plantea al hombre de hoy, en especial a quien tiene que combatir, un problema respecto a lo que durante siglos se ha denominado "patriotismo». La conciencia de pertenecer a una comunidad que hace posible interpretar la historia común y proyectar el futuro, constituye uno de los rasgos distintivos del ser humano. $\mathrm{Y}$ es sobre este sentimiento sobre el que está incidiendo la evolución sociopolítica del nuevo orden mundial, dificultando su comprensión y su valoración como virtud radical.

La Patria en cuanto concepto y realidad que se asume como valor colectivo y que, en consecuencia, provoca sentimientos de adhesión y de impulso a estar dispuestos a defenderla en caso necesario, se ve afectada por dos fuerzas dispares, que, sin negar expresamente su contenido, dificultan el desarrollo de aquél sentimiento. Una fuerza se orienta hacia la «mundialización» y otra a los «nacionalismos».

Como resultado de estas dos corrientes hoy existe una mayor dificultad para comprender y asumir en toda su profundidad lo que significa España, como quehacer común de todos los españoles, por encima de la estructura política que en cada momento histórico adopte el Estado. 
Por la primera se tiende hacia la Organización de estructuras supranacionales en los campos de la economía, la política, la defensa, etc, lo que diluye, en cierto sentido, el sentimiento de que formamos parte de una comunidad nacional, de una Patria común. Es importante tener en cuenta que durante siglos ha sido incuestionable este espíritu.

Por la segunda surgen los nacionalismos, en especial los excluyentes, que provocan la fragmentación de lo que se ha considerado como origen común y como razón de ser de los proyectos de futuro. El problema que pueden plantear a la consolidación de los nuevos Estados o a la construcción de Organizaciones supranacionales como la Unión Europea, ha de solucionarse por medios políticos y socioculturales. Solamente cuando se transforman en movimientos violentos o ilegales habrán de intervenir las Fuerzas de Seguridad del Estado y, en último término, las Fuerzas Armadas, pero siempre siguiendo las directrices que, en cada momento, adopte el Gobierno.

$\mathrm{Al}$ margen de estas misiones que las Fuerzas Armadas pudieran tener en algunos casos, los ejércitos, como elementos más específicos de la defensa, ejercen una indudable influencia en pro de la cohesión nacional, en la medida que la seguridad es un bien colectivo de la que se benefician todos los ciudadanos aunque no colaboren en ella (y hasta en el caso de que la rechacen). Además de estáaportación fundamental, los Ejércitos realizan un papel clave ya que precisamente por su carácter nacional facilitan la superación de los localismos y de las barreras regionalistas. Conviene recordar la evolución histórica del paso de los ejércitos vinculados al soberano, en definitiva a una persona, a los nacionales, en los que el conjunto de la sociedad pasó a ser el «propietario» y la razón de ser de su actividad.

De igual modo la participación en la defensa europea y en las Operaciones de Paz propiciadas por la ONU. contribuyen a que la sociedad, en su conjunto, se desarrolle en paz y pueda llevar a cabo iniciativas de cooperación y de ayuda que de otro modo no serían posibles.

En línea con esta aportación tiene importancia el principio de «acción conjunta», objeto de estas reflexiones. No se trata de plantear como un ejemplo a seguir porque en el terreno de la política rigen pautas diferentes, pero el espíritu de unidad de la defensa y en consecuencia el de los instrumentos que mas específicamente tienen esta misión, como son los ejércitos, propician y estimulan los esfuerzos que se llevan a cabo en este proceso. 


\section{Incidencia en el ámbito de la sociedad}

Mucho se ha discutido sobre el servicio militar obligatorio y su influencia en la educación de la juventud. A través de él muchos jóvenes conocieron el significado de palabras como Patria, España, o servicio. Pero también hay que reconocer que en los últimos años y por motivos que sería largo detallar, no sólo habían perdido este papel, sino que, en muchos casos, ha tenido el efecto contrario en relación a la defensa.

Tomada la decisión de profesionalizar el personal de tropa, no es momento de mirar hacia atrás con nostalgia, porque el efecto integrador de la Defensa puede, y debe, desarrollarse también con el nuevo modelo de Fuerzas Armadas. Importa poco que se haya perdido el carácter universal de la prestación del servicio militar, esto es, que obligue a todos los jóvenes sin distinción de clases sociales, ya que un gran número de jóvenes dedicarán voluntariamente varios años de su vida a la defensa de los intereses nacionales y este hecho tiene, por si mismo, un importante poder de integración.

Tampoco tiene porque restar fuerza a este efecto integrador el hecho de que muchos se lo planteen como una salida profesional, por cuanto la profesionalidad no merma el sentido positivo que tiene el espíritu de servicio, como bien queda demostrado en los cuadros de mando que desde hace muchos años han sido profesionales.

Por otro lado, la preparación que precisa el soldado moderno es mucho mas amplia y exigente que antaño. El combatiente del nuevo siglo necesitará una gran formación técnica para el mantenimiento y empleo de los medios tecnológicos, pero sobre todo habrá de poseer una sólida formación moral y gran equilibrio psicológico. Ha de ser capaz de combatir aislado o formando parte de grupos muy reducidos, habrá de integrarse con soldados de otros países y, sobre todo, habrá de tener una clara conciencia de porqué arriesga su vida.

Los Ejércitos también contribuyen positivamente a través de su colaboración con otras Instituciones. En especial con Protección civil, cooperan a solucionar problemas que no son propiamente militares y de estas actividades suelen nacer relaciones que van mas allá del problema concreto que los convocó. Es conocida la colaboración de Unidades en la extinción de incendios y en paliar los efectos de las grandes catástrofes, como inundaciones, terremotos, etc, pero también son de destacar las labores de salvamento, en tierra y mar, el transporte de órganos para trasplantes y una larga serie de misiones similares. 
Esta cooperación no queda limitada a los casos de carácter humanitario, sino que también hay que destacar su aportación en la organización de grandes eventos como son las Olimpiadas, los Campeonatos deportivos internacionales o las grandes concentraciones que se producen con ocasión de Congresos, Encuentros de jóvenes, etc. En todos ellos tienen un papel fundamental, aunque lo realicen siempre con carácter subsidiario.

Su carácter es subsidiario en cuanto que la sociedad puede, o debe, tener otros medios y organismos para hacer frente a estos problemas, pero ello no resta importancia a la labor realizada. Por otro lado, el ejemplo de naciones mas desarrolladas demuestra que aún teniendo medios de Protección social, debidamente dotados y organizados, surgen las ocasiones en las que las Fuerzas Armadas son la Institución mas capacitada para solucionar el problema planteado.

En este sentido es significativo que en la Doctrina OTAN se incluyan muchas misiones de este tipo, dando gran importancia a la colaboración con las Organizaciones no gubernamentales (ONGS). El acelerado incremento de ONGS, de carácter nacional o internacional, constituye uno de los fenómenos sociales mas notables de las últimas décadas. A pesar de los problemas que en ocasiones plantean sus actividades, en muchos casos son las organizaciones con mayor capacidad para intervenir en determinadas situaciones y paliar problemas difíciles de abordar desde instancias oficiales. Son muchos los Gobiernos que parte de sus programas de cooperación los canalizan a través suya y en varios de los recientes conflictos han demostrado su eficacia.

Eficacia que se ve multiplicada cuando se establecen cauces de cooperación con Fuerzas de Paz y que, en bastantes casos, precisan para ser eficaces de un entorno de seguridad que sólo le pueden proporcionar las unidades militares. En la medida que se fomente la colaboración mutua se logrará un mejor aprovechamiento de los medios, humanos y materiales, empleados en una zona de conflicto.

También aquí hay que señalar que el conocimiento de estas operaciones y la mejora de las relaciones del conjunto de la sociedad con sus Fuerzas Armadas y de éstas con la sociedad, se ha de traducir en un salto adelante en el desarrollo del espíritu de convivencia y en la conjunción de los esfuerzos de los diferentes sectores. Las Reales Ordenanzas para las Fuerzas Armadas ${ }^{8}$ en varios de sus artículos insisten en este tema y recalcan la necesidad de que los Ejércitos como Institución y los militares como individuos han de sentirse integrados y respaldados por el pueblo, cuya defensa es la que en definitiva justifica su existencia. 


\section{Incidencia en el ámbito de los valores}

Son muchas las voces que desde distintos sectores advierten que nos encontramos en un período de crisis de valores y que es necesario dar respuesta al reto que supone para la Humanidad el progreso de las últimas décadas. Es importante, en consecuencia, participar en el esfuerzo colectivo que debe hacer la sociedad para equilibrar el proceso de desarrollo material, con uno equivalente en el ámbito de los valores.

En este sentido las Fuerzas Armadas tienen mucho que aportar. Es común a todas las naciones occidentales que en el proceso educativo de sus cuadros de mando y del personal de tropa, tenga la Etica un papel destacado. $\mathrm{Y}$ ello no se debe a la pretensión de distinguirse de otros sectores, sino que el ejercicio de la profesión militar exige tener asumidos determinados esquemas de valores. Es de destacar que, en España, las Fuerzas Armadas son la única Institución que tiene, con rango de Ley, un Código moral de conducta. Las Reales Ordenanzas ya citadas (sancionadas el 28 de diciembre de 1978), recogen preceptos de Ordenanzas anteriores, pero fundamentalmente han incorporado valores que son mas necesarios hoy que nunca.

En el marco de estas ideas cobra importancia el hecho de que los valores militares son ante todo integradores. Cabría pensar en una Ética «egocéntrica», pero la realidad es que las necesidades del hombre tienen, como referencia permanente, el resto de la comunidad, sea la familiar, la del trabajo o la mas amplia de la nación. Es por ello que el código ético vigente en el estamento militar tiene aplicación en el entorno y, en todo caso, puede suponer una aportación importante.

Como ejemplo cabe citar el enriquecimiento que para la acción de conjunto supone la importancia que en el ámbito castrense tiene el compañerismo. O el alcance del patriotismo que hoy se ha de relacionar con la participación en la defensa de los intereses colectivos, propios y los de las otras naciones. En definitiva en lograr un espacio de paz y seguridad internacionales. Todos estos valores pertenecen al conjunto de la nación y los Ejércitos contribuyen a fomentarlos en la medida que se encuentran integrados en la sociedad y actúan conjuntamente con ella.

La conveniencia de que la Etica recuperase el papel preponderante que tuvo en otros tiempos se ha transformado en una necesidad imperiosa. Los acelerados cambios sociales y la aparición de nuevas tecnologías que permiten al hombre adentrarse en campos hasta ahora inimaginables (piensese en los avances en Genética, los trasplantes 
de órganos, los viajes interplanetarios o la capacidad de modificar el equilibrio ecológico), apremian a que se recupere el sentido ético como uno de los rasgos característicos de la sociedad postmoderna.

\section{A modo de conclusiones}

Sería un error plantear un resumen de las ideas expuestas porque, inevitablemente, se perdería su hilazón y se puede caer en la tentación de finalizar con una «moraleja». En un tema tan complejo como el de la «acción conjunta» o la conjunción de esfuerzos dentro de las Fuerzas Armadas, necesariamente se han dejado puntos sin tratar y, en algunos casos, se han razonado de modo insuficiente. En consecuencia la primera conclusión es que ha de interpretarse como una reflexión en voz alta, a la que es necesario incorporar los datos y las ideas planteadas en otros trabajos.

Sobre la base de que el hombre primitivo ya conoció la necesidad de cazar y trabajar en grupo, se ha insistido que la llamada «acción conjunta» es mucho mas que la simple extensión de aquella necesidad. Se trata de un salto cualitativo ya que plantea un modelo diferente de entender la Defensa.

En este sentido se debe estudiar desde dos contextos distintos: sobre el del propio ser de las FAS., y sobre el del objeto que tiene la defensa en una nación. En relación al «ser» lo mas importante no es la evolución histórica de los Ejércitos, sino que se ha llegado a un punto en el que, para las naciones desarrolladas de Occidente, la Defensa ha de entenderse como un proyecto colectivo. De ahí la importancia, entre otras, del desarrollo de la interoperatividad y el de las características de las Unidades modernas.

En relación al objeto de la Defensa, lo mas importante es destacar que, por sí misma, tiene un efecto integrador en la sociedad, tanto porque no se puede concebir como una acción parcelada, en lo geográfico o en lo humano, como por su colaboración con el resto de la sociedad en las misiones de apoyo.

Por último conviene reflexionar sobre la crisis de valores en la que parece se encuentra la sociedad moderna. Desde hace siglos los Ejércitos poseen un código moral que fue aprobado como ley y por tanto como expresión de lo que el pueblo español desea. Las Reales Ordenanzas son pues de aplicación no sólo a las FAS. sino también pueden servir como referencia a la sociedad en general. 
De lo expuesto se deduce que la «acción conjunta» va mucho mas allá que la simple conjunción de esfuerzos y sin que sea del todo correcto considerarla como un rasgo más que demuestra la tendencia al «punto omega» que, según Tehilard de Chardin, rige el cosmos, ha de entenderse como un factor que coincide con esta fuerza y, en consecuencia, que interviene a favor de la evolución de la historia.

\section{Notas}

1 Sun Tzu. Los 13 Artículos de la Guerra. Ed. Defensa. Resulta mas completo el trabajo del TCOL. E. Cholet «El arte militar de los chinos». Ed. Pleamar.

2 Clausewitz. De la Guerra. Ed. Defensa.

3 Luis F. Rey ARRoyo. La actuación conjunta en los ejércitos del 2000. Revista Española de Defensa n..$^{\circ} 136$.

4 Doctrina para el empleo de la Fuerza Terrestre. Texto oficial. La palabra «doctrina» se emplea para designar un Reglamento que tiene especiales características, ya que constituye el fundamento del los restantes Reglamentos y Orientaciones.

5 Revista de Aeronáutica. Julio de 1981. Publicó un Dossier sobre el tema.

6 Cte. Juan M. Ga MonTAÑo. «La interoperatividad». Rev. Ejército Sep. 1993.

7 Existe cierta confusión con las siglas IESD (Identidad Europea de Seguridad y Defensa) y PESC (Política Europea de Seguridad Común). Aunque corresponden a distintas iniciativas coinciden en el objetivo.

8 Ley 85/1978. Reales Ordenanzas para las Fuerzas Armadas. 